\title{
Impact of Abnormal Glucose Tolerance, Hypertension and Other Risk Factors on Coronary Artery Disease
}

\author{
Yosuke Nakamura, MD; Shigeyuki Saitoh, MD; Satoru Takagi, MD; Hirofumi Ohnishi, MD; \\ Yu Chiba, MD; Nobuo Kato, MD; Hiroshi Akasaka, MD; Tetsuji Miura, MD; \\ Kazufumi Tsuchihashi, MD; Kazuaki Shimamoto, MD
}

\begin{abstract}
Background The degree to which abnormal glucose tolerance contributes to the development of coronary artery disease (CAD) has not been clarified in Japanese. The relationship between abnormal glucose tolerance and severity of coronary artery stenosis, as well as the contributions of hypertension, diabetes and other risk factors for CAD to recurrence of the disease, were investigated in the present study.

Methods and Results The subjects were 474 consecutive patients (mean age: $63.8 \pm 11.3$ years) with suspected CAD who were admitted to Sapporo Medical University Hospital during April 1, 1997 to March 31, 2004. The coronary index and stenosis score were higher in subjects with diabetes mellitus (DM) and in subjects with impaired fasting glucose (IFG) and impaired glucose tolerance (IGT) than in subjects with normal glucose tolerance (NGT). Ischemic episodes recurred during the observation period (mean 2.5 years) in 61 of 341 patients diagnosed as having CAD. In the follow-up subjects, systolic blood pressure (SBP) was significantly higher in the recurrence group than in the non-recurrence group, and SBP was a significant variable in logistic regression analysis after adjustment for age, gender, hemoglobin A1c, total cholesterol, body mass index, smoking history, family history and stenosis score. The relative risk of recurrence became 1.7 -fold higher with a rise in SBP of $10 \mathrm{mmHg}$ (95\% confidence interval: 1.252-2.250). Analysis of the relationship between glucose tolerance and recurrence showed that the rate of recurrence was higher in patients with IFG+IGT + DM than in those with NGT.

Conclusions CAD progresses not only in patients with DM but also in those with IGT. The rate of recurrence of ischemic episodes increases in individuals with IGT or DM, and suggesting that hypertension is a risk factor for recurrence of ischemic episodes. Management of glucose tolerance and blood pressure is therefore important for prevention of CAD in Japanese. (Circ J 2007; 71: 20-25)
\end{abstract}

Key Words: Coronary artery disease; Impaired glucose tolerance; Recurrence

$\mathbf{T}$ here is concern about the reported dramatic increases in recent years in the incidences of diabetes mellitus (DM) and impaired glucose tolerance (IGT) in Japanese ${ }^{1-3}$ and it is known that coronary artery disease $(\mathrm{CAD})$ is severe in diabetic patients and they have a poor prognosis-7

There have also been reports on the contribution of abnormal glucose tolerance, including impaired fasting glucose (IFG) and IGT, to the development of $\mathrm{CAD}^{8-11}$ but there have been only a few reports on the impact of glucose intolerance on severity of coronary artery stenosis determined by coronary angiography!2,13 Therefore, using patients admitted to Sapporo Medical University Hospital as subjects, we investigated the relationship between abnormal glucose tolerance and severity of coronary artery stenosis in a cross-sectional study, and we also investigated the contributions of insulin resistance (IR) and various risk factors for CAD, including hypertension (HT), DM, IGT, hyperlipidemia and obesity, to recurrence of ischemic episodes in a longitudinal study.

(Received January 19, 2006; revised manuscript received September 21, 2006; accepted October 13, 2006)

Second Department of Internal Medicine, Sapporo Medical University, Sapporo, Japan.

Mailing address: Yosuke Nakamura, MD, Second Department of Internal Medicine, Sapporo Medical University, W-16, S-1, Chuo-ku, Sapporo 060-8543, Japan. E-mail: yosuken@sapmed.ac.jp

\section{Methods}

\section{Study Population}

Relationship Between Abnormal Glucose Tolerance and Severity of Coronary Artery Stenosis (Cross-Sectional Study) The subjects in the cross-sectional study were 474 consecutive patients (mean age: $63.8 \pm 11.3$ years) who were admitted to Sapporo Medical University Hospital for investigation of chest pain and suspected CAD during the period from April 1, 1997 to March 31, 2004 and who were subsequently diagnosed as having $\mathrm{CAD}$, based on findings from coronary angiography, nuclear medical examinations, and exercise and 12-lead electrocardiogram. Patients who had complications such as heart valve disease, pulmonary thromboembolism, aortic dissection, myocarditis, and pericarditis were excluded from the subjects. Of the 474 patients, 224 had acute coronary syndrome, including acute myocardial infarction and unstable angina, 148 had effort angina pectoris, 64 had silent myocardial ischemia, and 38 had vasospastic angina pectoris.

Relationships Between Risk Factors and Recurrence of Ischemic Episodes (Longitudinal Study) Of the subjects who had been diagnosed as having CAD during their first admission and who had been discharged after successful revascularization and chest pain control had been achieved by percutaneous coronary intervention $(\mathrm{PCI})$, coronary artery bypass grafting (CABG) and drug therapy, the recurrence group was defined as those who had chest pain be- 
Table 1 Comparison of the Characteristics of the NGT, IFG + IGT, and DM Groups

\begin{tabular}{|c|c|c|c|c|}
\hline & $\begin{array}{c}N G T \\
(n=237)\end{array}$ & $\begin{array}{c}I F G+I G T \\
(n=65)\end{array}$ & $\begin{array}{c}D M \\
(n=172)\end{array}$ & $p$ value \\
\hline Age (years) & $63.6 \pm 11.7$ & $66.6 \pm 9.6$ & $64.9 \pm 10.6$ & $N S$ \\
\hline$F / M$ & $69 / 168$ & $17 / 48$ & $43 / 129$ & NS \\
\hline$F P G(m g / d l)$ & $92.6 \pm 11.0$ & $103.1 \pm 12.7$ & $124.2 \pm 33.0$ & $<0.001$ \\
\hline Hemoglobin Alc (\%) & $5.1 \pm 0.4$ & $5.3 \pm 0.4$ & $6.6 \pm 1.3$ & $<0.001$ \\
\hline$S B P(m m H g)$ & $120.7 \pm 15.0$ & $120.9 \pm 15.1$ & $126.5 \pm 16.3$ & 0.001 \\
\hline$D B P(m m H g)$ & $69.4 \pm 8.9$ & $71.2 \pm 9.0$ & $71.4 \pm 8.9$ & $N S$ \\
\hline$T C(m g / d l)$ & $183.6 \pm 26.6$ & $183.9 \pm 33.5$ & $182.2 \pm 34.7$ & $N S$ \\
\hline$T G(m g / d l)$ & $132.2 \pm 55.7$ & $135.0 \pm 63.4$ & $147.1 \pm 92.7$ & $N S$ \\
\hline$H D L-C(m g / d l)$ & $44.8 \pm 14.3$ & $43.1 \pm 15.3$ & $42.4 \pm 12.5$ & $N S$ \\
\hline$L D L-C(m g / d l)$ & $113.3 \pm 24.0$ & $113.6 \pm 27.0$ & $112.0 \pm 30.4$ & $N S$ \\
\hline$B M I\left(\mathrm{~kg} / \mathrm{m}^{2}\right)$ & $23.9 \pm 3.1$ & $24.4 \pm 3.2$ & $24.9 \pm 4.1$ & 0.02 \\
\hline Smoking (\%) & 55.6 & 61.8 & 56.7 & $N S$ \\
\hline Family history (\%) & 15.7 & 34.4 & 21.5 & 0.004 \\
\hline Drinking (\%) & 38.6 & 42.9 & 40.2 & $N S$ \\
\hline \multicolumn{5}{|l|}{ Drug therapy (\%) } \\
\hline$A C E I$ or $A R B$ & 57.2 & 51.5 & 63.2 & $N S$ \\
\hline$\beta$-blocker & 43.2 & 53.0 & 56.7 & 0.022 \\
\hline Ca-antagonist & 44.5 & 43.9 & 45.6 & $N S$ \\
\hline Diuretics & 8.1 & 16.7 & 12.9 & $N S$ \\
\hline$H M G-C o A$ & 44.5 & 57.6 & 48.5 & $N S$ \\
\hline
\end{tabular}

Smoking, family history, drinking, drug therapy are shown by percentage of positive for these factors.

Other factors are shown by mean $\pm S D$.

$A C E I$, angiotensin-converting enzyme inhibitor; ARB, angiotensin-receptor blocker; HMG-CoA, HMG-CoA reductase inhibitor.

See text for other abbreviations.

cause of ischemia during their second admission and for whom results of exercise tolerance tests, nuclear medical examinations and imaging examinations carried out regularly on an out-patient basis confirmed ischemia.

In order to compare the data for patients with recurrence of myocardial ischemia and patients without recurrence, we carried out a longitudinal study using 341 subjects (mean age: $63.2 \pm 12.0$ years) selected from those in the crosssectional study for whom follow-up was possible. There was no difference between items evaluated in the subjects during admission in the cross-sectional study and the follow-up subjects.

\section{Study Protocol}

Cross-Sectional Study Coronary angiography was performed on 380 of the 474 subjects in the cross-sectional study and based on the results stenosis was scored according to the American Heart Association functional classification: ${ }^{14} 5$ points for $99-100 \%$ stenosis, 4 for $90 \%, 3$ for $75 \%, 2$ for $50 \%, 1$ for $25 \%$ and 0 for $0 \%$. The maximum coronary stenosis point in each case in the left anterior descending artery, left circumflex artery, or right coronary artery was defined as the coronary index ${ }^{14}$ and the stenosis score $^{14}$ was calculated as the sum of the stenosis points of each branch.

The following items were measured in the early morning after overnight fasting during admission: body mass index (BMI), systolic blood pressure (SBP), diastolic blood pressure (DBP), fasting plasma glucose (FPG), hemoglobin ( $\mathrm{Hb}$ ) A1c, total cholesterol (TC), triglyceride (TG), and high-density lipoprotein-cholesterol (HDL-C). Low-density lipoprotein-cholesterol (LDL-C) was calculated by Friedewald's formula! 15

Subjects who showed symptoms of DM or who had a casual plasma glucose (PG) level $\geq 200 \mathrm{mg} / \mathrm{dl}, \mathrm{FPG} \geq 126 \mathrm{mg} / \mathrm{dl}$ or $2 \mathrm{~h}-\mathrm{PG}$ during a 75 -g oral glucose tolerance test (OGTT) $\geq 200 \mathrm{mg} / \mathrm{dl}$ were diagnosed as having DM according to the ADA criteria! ${ }^{16}$ Subjects who had $2 \mathrm{~h}-\mathrm{PG} \geq 140 \mathrm{mg} / \mathrm{dl}$ and
$2 \mathrm{~h}-\mathrm{PG}<200 \mathrm{mg} / \mathrm{dl}$ were diagnosed as having IGT, and those who had FPG $\geq 110 \mathrm{mg} / \mathrm{dl}$ and FPG $<126 \mathrm{mg} / \mathrm{dl}$ were diagnosed as having IFG.

Subjects with a family history of CAD were defined as those with any family member within 2 generations having CAD. Subjects with a smoking habit were defined as current smokers who smoked 1 or more cigarettes per day. Subjects with a drinking habit were defined as all of those who drank other than those who drank only on special occasions.

Data obtained at the time of discharge were used for analysis of the relationship between glucose tolerance and severity of coronary artery stenosis.

Longitudinal Study The endpoint of the longitudinal study was recurrence of ischemic episodes during the observation period, which was 2.4 years.

Items evaluated during admission in this series of study were the same as those in the cross-sectional study. Relationships between recurrence of ischemic episodes and coronary risk factors, including HT, abnormal glucose tolerance, hyperlipidemia and obesity, were analysed. Because the number of follow-up subjects with IGT $(n=25)$ was small, we divided the follow-up subjects into normal glucose tolerance (NGT) and IFG+IGT + DM groups for comparison of the rates of recurrence.

Eighty-five patients underwent 75-g OGTT, and those who had an insulin concentration $2 \mathrm{~h}$ after glucose loading (120 min IRI) $\geq 64 \mathrm{mU} / \mathrm{L}$ were defined as the IR group ${ }^{17} \mathrm{We}$ compared the rates of ischemic episodes recurrence in the IR group and non-insulin resistance (NR) group to determine whether IR is associated with recurrence of ischemic episodes.

\section{Statistical Analysis}

Data are expressed as means \pm standard deviation. Differences between mean values in 2 groups were examined by the unpaired t-test. One-way ANOVA was used to examine differences between mean values in the 3 groups (NGT, 
Table 2 Comparison of the Angiographic Characteristics of the NGT, IFG + IGT, and DM Groups

\begin{tabular}{lcccc}
\hline \hline & $\begin{array}{c}\text { NGT } \\
(n=190)\end{array}$ & $\begin{array}{c}\text { IFG }+I G T \\
(n=53)\end{array}$ & $\begin{array}{c}\text { DM } \\
(n=137)\end{array}$ & $p$ value \\
\hline No. of diseased vessels & $1.39 \pm 1.01$ & $1.77 \pm 0.81$ & $2.22 \pm 0.78^{*}$ & $<0.001$ \\
Coronary index & $4.01 \pm 1.67$ & $4.77 \pm 0.53^{*}$ & $4.73 \pm 0.52^{*}$ & $<0.001$ \\
Stenosis score & $6.96 \pm 4.05$ & $8.73 \pm 3.52^{*}$ & $10.18 \pm 3.01^{*}$ & $<0.001$ \\
\hline
\end{tabular}

*vs NGT $p<0.05$.

See text for abbreviations.

Table 3 Clinical Characteristic of the Groups With Recurrence of Ischemic Episodes and Without

\begin{tabular}{|c|c|c|c|}
\hline & $\begin{array}{c}\text { Recurrence } \\
(-) \\
(n=280)\end{array}$ & $\begin{array}{c}\text { Recurrence } \\
(+) \\
(n=61)\end{array}$ & $p$ value \\
\hline Age (years) & $64.1 \pm 11.5$ & $64.0 \pm 10.6$ & $N S$ \\
\hline$N(F / M)$ & $72 / 208$ & $16 / 45$ & $N S$ \\
\hline$F P G(m g / d l)$ & $107.8 \pm 29.7$ & $102.1 \pm 22.5$ & $N S$ \\
\hline Hemoglobin A1c (\%) & $5.8 \pm 1.3$ & $5.7 \pm 0.8$ & $N S$ \\
\hline$S B P(m m H g)$ & $120.1 \pm 17.0$ & $131.5 \pm 5.0$ & $<0.001$ \\
\hline$D B P(m m H g)$ & $70.0 \pm 9.3$ & $72.0 \pm 8.2$ & $N S$ \\
\hline$T C(m g / d l)$ & $185.9 \pm 31.5$ & $177.0 \pm 27.1$ & 0.039 \\
\hline$T G(m g / d l)$ & $140.5 \pm 78.6$ & $134.1 \pm 57.2$ & $N S$ \\
\hline$H D L-C(m g / d l)$ & $42.6 \pm 12.9$ & $45.7 \pm 16.0$ & NS \\
\hline$L D L-C(m g / d l)$ & $114.8 \pm 28.2$ & $110.0 \pm 27.0$ & $N S$ \\
\hline$B M I\left(\mathrm{~kg} / \mathrm{m}^{2}\right)$ & $24.1 \pm 3.5$ & $25.2 \pm 3.5$ & 0.022 \\
\hline Smoking (\%) & 59.6 & 56.1 & $N S$ \\
\hline Family history (\%) & 19.4 & 16.4 & $N S$ \\
\hline Drinking (\%) & 37.0 & 36.2 & $N S$ \\
\hline \multicolumn{4}{|l|}{ Drug therapy (\%) } \\
\hline$A C E I$ or $A R B$ & 63.3 & 59.3 & $N S$ \\
\hline$\beta$-blocker & 47.7 & 71.2 & 0.001 \\
\hline Ca-antagonist & 37.7 & 39.0 & $N S$ \\
\hline Diuretics & 10.7 & 13.6 & NS \\
\hline$H M G-C o A$ & 47.7 & 52.5 & $N S$ \\
\hline
\end{tabular}

See Table 1 for abbreviations.

Values are mean $\pm S D$. Recurrence; ischemic episodes recurrence.

Smoking, family history, drinking, drug therapy are shown by percentage of positive for these factors.

IFG + IGT, and DM), and multiple logistic regression analysis was used to examine relations between ischemic episode recurrence and other risk factors. A p-value of less than 0.05 in these analyses was considered significant. All statistical analyses were performed using SPSS version 12.0J (Chicago, IL, USA).

\section{Results}

Relationship Between Abnormal Glucose Tolerance and Severity of Coronary Artery Stenosis (Cross-Sectional Study)

Table 1 shows a comparison of data in the NGT, IFG+ IGT and DM groups. The percentage of subjects with a family history of CAD was significantly higher in the IFG + IGT group than in the other 2 groups.

Table 2 shows a comparison of the coronary artery lesions in the NGT, IFG+IGT and DM groups. Both the IFG + IGT and DM groups showed higher values than the NGT group for number of diseased vessels (NGT vs IFG + IGT vs DM: $1.39 \pm 1.01$ vs $1.77 \pm 0.81$ vs $2.22 \pm 0.78$; $\mathrm{p}<$ $0.001)$, coronary index $(4.01 \pm 1.67$ vs $4.77 \pm 0.53$ vs $4.73 \pm$ $0.52 ; \mathrm{p}<0.001)$ and stenosis score $(6.96 \pm 4.05$ vs $8.73 \pm 3.52$ vs $10.18 \pm 3.01 ; \mathrm{p}<0.001)$, indicating that $\mathrm{CAD}$ progresses not only in those with frank DM but also at the stage of IFG+IGT.
Table 4 Multiple Logistic Regression Analysis for Ischemic Episodes Recurrence

\begin{tabular}{lccc}
\hline \hline & Odds ratio & p value & $95 \%$ CI \\
\hline SBP & 1.678 & 0.001 & $1.252-2.250$ \\
Age & 0.983 & 0.399 & $0.946-1.023$ \\
Sex & 0.910 & 0.852 & $0.339-2.441$ \\
TC & 0.994 & 0.464 & $0.977-1.011$ \\
BMI & 1.134 & 0.037 & $1.008-1.276$ \\
Hemoglobin A1c & 0.737 & 0.122 & $0.501-1.085$ \\
Family history & 0.606 & 0.383 & $0.197-1.866$ \\
Stenosis score & 1.103 & 0.166 & $0.960-1.267$ \\
Smoking & 0.882 & 0.780 & $0.366-2.128$ \\
\hline
\end{tabular}

Independent variables: ischemic episodes recurrence.

Dependent variables: SBP, Age, Sex, TC, BMI, Hemoglobin Alc, stenosis score, smoking, family history.

$S B P$, rise in $S B P$ of $10 \mathrm{mmHg}$.

CI, confidence interval. See text for other abbreviations.

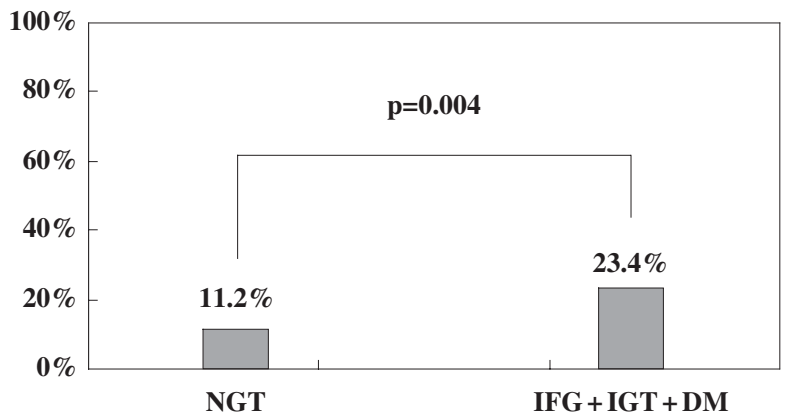

Fig 1. Comparison of ischemic episodes recurrence rate between normal glucose tolerance (NGT), impaired fasting glucose (IFG) + impaired glucose tolerance (IGT) + diabetes mellitus (DM) groups.

\section{Relationships Between Risk Factors and Recurrence of Ischemic Episodes (Longitudinal Study)}

Ischemic episodes recurred in 61 of the 341 subjects during the observation period. Table 3 shows a comparison of risk factors in subjects with recurrence of ischemia and those without the recurrence. SBP and BMI were significantly higher in the recurrence group than in the non-recurrence group.

Table 4 shows the results of multiple logistic regression analysis using ischemic episode recurrence as an independent variable. When adjustments were made for age, gender, HbA1c, TC, BMI, smoking history, family history and stenosis score, SBP became a significant variable for recurrence, and the relative risk of recurrent ischemia was 1.7 -fold higher with a rise in SBP of $10 \mathrm{mmHg}$. The rates of ischemic episode recurrence for the NGT and IFG+IGT+ DM groups are shown in Fig 1. The rate of recurrence was higher in the IFG+IGT+DM group $(23.4 \%)$ than in the NGT group $(11.2 \%)(\mathrm{p}=0.004)$. When we defined IR according to the plasma insulin level $2 \mathrm{~h}$ after glucose loading 
as stated before, the rate of recurrence of ischemia in the IR group was higher than that in the NR group (Fig 2) (NR vs IR: $4.8 \%$ vs $40.0 \%, p=0.009$ ). Although not shown in the table, the HOMA-IR value, an index of IR, was higher, though not significantly, in the recurrence group than in the non-recurrence group (recurrence group vs non-recurrence group: 2.1 vs $1.7, \mathrm{p}=0.372$ ).

\section{Discussion}

Relationship Between Abnormal Glucose Tolerance and Severity of Coronary Artery Stenosis (Cross-Sectional Study)

The subjects with DM in this study also showed stenosis in many coronary artery branches and severe stenosis. Diabetes is often complicated with CAD, and it has been shown that diabetes also has a significant untoward effect on the prognosis and severity of CAD. Our findings agree with the results of a study by Dortimer et al showing that many patients with diabetes had stenosis in 3 branches of the coronary artery, 18 and the results of a study by Kip et al showing extensive diffuse coronary arteriosclerosis in diabetics with CAD ${ }^{19}$

The results of the present study indicate that not only subjects with diabetes but also those with abnormal glucose tolerance have more severe stenosis and stenosis in more vessels than subjects with NGT. However, the association of abnormal glucose tolerance with severity of CAD is not fully characterized!2,13 The study by Kataoka et al showed a significant correlation between IGT and severity of $\mathrm{CAD}^{12}$ and our results agree with their finding. However, the study by Horimoto et al suggested that there was no significant difference between the severity of angiographic coronary atherosclerosis in subjects with NGT and IGT $!^{3}$ This discrepancy may be related to the small numbers of subjects, and differences in the characteristics of the subjects entered in the study protocols. Nevertheless, further investigation of this issue is needed.

It has been shown that individuals with abnormal glucose tolerance have IR in addition to a high PG level. Currently, whether hyperglycemia in IGT patients or IR are responsible for the IGT-CAD association has been a subject of investigation. Recently, Satoh et $\mathrm{al}^{20}$ and Takezako et $\mathrm{al}^{21}$ have reported that IR rather than hyperglycemia is associated with the severity of CAD. In our study, we examined the relationships between number of diseased vessels, $2 \mathrm{~h}-\mathrm{PG}$ and $120 \mathrm{~min}$ IRI using subjects other than those with DM. We found that subjects with multiple vessel disease had higher values for both $2 \mathrm{~h}-\mathrm{PG}$ (patients with multiple vessel disease vs patients with 1 diseased vessel or less: $139.3 \pm$ $27.1 \mathrm{mg} / \mathrm{dl}$ vs $116.5 \pm 21.2 \mathrm{mg} / \mathrm{dl} ; \mathrm{p}<0.001)$ and $120 \mathrm{~min}$ IRI $(92.3 \pm 58.7 \mathrm{mU} / \mathrm{L}$ vs $67.6 \pm 39.1 \mathrm{mU} / \mathrm{L} ; \mathrm{p}=0.05)$ compared with subjects with 1 diseased vessel or less. This suggests that both postprandial hyperglycemia and hyperinsulinemia are involved in the development of CAD in patients with IGT.

IR results in compensatory hyperinsulinemia, which may give rise to an accumulation of risk factors for CAD, provoking a direct mechanism for the progression of arteriosclerosis. It has, in fact, been reported that subjects with IR have a high incidence of cardiovascular disease ${ }^{22}$ and show progression of $\mathrm{CAD}^{23,24}$ and our results also suggest that IR is involved in the progression of CAD. We previously reported that $\mathrm{CAD}$ in IR is characterized by stenosis of multiple coronary artery branches and peripheral lesions 24

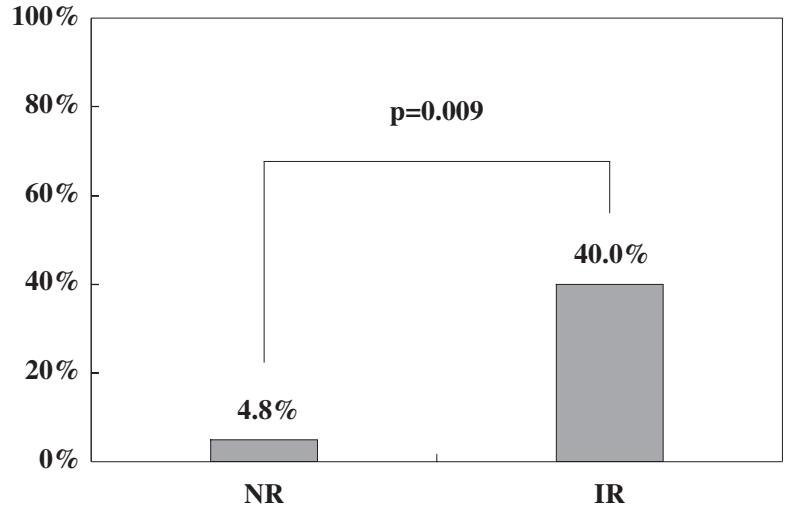

Fig 2. Comparison of ischemic episodes recurrence rate between non-insulin resistance (NR) and insulin resistance (IR).

Not only the accumulation of risk factors for CAD but also a reduction in blood flow in the coronary artery occur in a state of IR, 25 and it is possible that these factors, acting either alone or synergistically, cause progression of CAD.

\section{Relationships Between Risk Factors and Recurrence of Ischemic Episodes (Longitudinal Study)}

There was no difference between FPG or lipid levels in the recurrence group and non-recurrence group, but SBP and BMI were significantly higher in the recurrence group. We found in the present study that SBP contributed significantly to recurrence of ischemic episodes, even when adjustments were made for age, gender, HbAlc, TC, BMI, smoking history, family history and stenosis score, and that the relative risk of recurrence was 1.7 -fold higher with a rise in $\mathrm{SBP}$ of $10 \mathrm{mmHg}$. Although there have been only a few studies on the relationship between blood pressure and recurrence of ischemic episodes, Xiao-Fei Zhang et al $^{26}$ found that higher SBP increases the mortality from cardiovascular disease in Chinese subjects, and our findings agree with their report. In the present study, there was no significant difference between the percentages of subjects receiving hypotensive drugs in the non-recurrence group (90.0\%) and recurrence group (93.2\%). There was also no significant difference between the 2 groups in the percentages of subjects taking an angiotensin-converting enzyme inhibitor, an angiotensin-receptor blocker, a $\mathrm{Ca}^{2+}$ antagonist or a diuretic drug, suggesting that high blood pressure has a direct effect on recurrence of ischemic episodes.

It has been reported that obesity is involved in the onset of $\mathrm{CAD}^{27,28}$ and in the present study BMI was found to be significantly higher in the recurrence group, which is consistent with the recently proposed concept of metabolic syndrome in which abdominal obesity is an important risk factor for cardiovascular disease.

The rate of recurrence of ischemic episodes was higher in subjects with IFG+IGT+DM than in subjects with NGT. However, no significant difference was found between the levels of FPG or HbA1c or the frequencies of use of hypoglycemic drugs or insulin in the recurrence and non-recurrence groups. On the other hand, analysis of the effect of IR, assessed by the insulin concentration $2 \mathrm{~h}$ after glucose loading, ${ }^{17}$ showed that the rate of recurrence was significantly higher in the IR group than in the NR group. These results suggest that IR rather than hyperglycemia increases the risk of recurrence of ischemic episodes, which agrees with an earlier study ${ }^{29}$ showing association of IR with coro- 
nary restenosis after PCI.

Lipid profile did not differ between the recurrence group and the non-recurrence group except for TC, which was significantly lower in the recurrence group, presumably because of the strict control of TC level in the subjects during their hospitalization. The proportion of subjects taking cholesterol-lowering drugs was approximately $50 \%$ in both groups.

\section{Study Limitations}

This study has 3 limitations. First, subjects were hospitalized at least once, and in many of the cases, CAD was severe. Second, the number of subjects was relatively small. Further investigation using a larger number of subjects is necessary to confirm the present observation. The third limitation concerns recurrence. In this study, the recurrence group of subjects was defined as those who had been diagnosed as having CAD during their first admission and who had been discharged after receiving PCI, CABG and drug therapy and then had chest pain because of ischemia, which was confirmed by results of exercise tolerance tests, nuclear medical examinations and imaging examinations carried out regularly on an outpatient basis, during their second admission. The methods used for diagnosis and the treatment methods differed among the subjects, and the criteria for diagnosis of recurrence were not uniform. However, data for all of the subjects included in the recurrence group were used for analysis because exclusion of subjects not strictly meeting the criteria would have resulted in a very small number of subjects. It is also possible that the mechanisms of restenosis and new stenosis of the coronary artery are different, and the risk factors for new stenosis might not always be involved in restenosis. However, in this study, there were few cases of confirmed new stenosis, and some of the cases of confirmed new stenosis also included restenosis. It has also been reported that the rate of restenosis is high in cases of CAD complicated with DM. In this study, we therefore did not differentiate restenosis and new stenosis in our analysis of the effects of risk factors on stenosis.

In conclusion, our study revealed that CAD progresses at the stage of abnormal glucose tolerance and that the rate of ischemic episode recurrence increases in individuals with abnormal glucose tolerance, DM and HT. Therefore, it is important to manage and control glucose tolerance, IR and blood pressure for prevention of CAD.

\section{References}

1. Sekikawa A, Eguchi H, Tominaga M, Igarashi K, Abe T, Manaka H, et al. Prevalence of type 2 diabetes mellitus and impaired glucose tolerance in a rural area of Japan: The Funagata diabetes study. $J$ Diabetes Complications 2000; 4: 78-83.

2. Ohmura T, Ueda K, Kiyohara Y, Kato I, Iwamoto H, Nakayama K, et al. Prevalence of type 2 (non-insulin-dependent) diabetes mellitus and impaired glucose tolerance in the Japanese general population: The Hisayama Study. Diabetologia 1993; 36: 1198-1203.

3. Nakano M, Saitoh S, Takagi S, Fujisawa J, Aoyama S, Shimamoto $\mathrm{K}$, et al. Prevalence of glucose intolerance in Japan: From the National Circulatory Disorders Basic Survey, 1990. Nippon Ronen Igakkai Zasshi 1998; 35: 839-844.

4. Haffner SM, Lehto S, Ronnemaa T, Pyorala K, Laakso M. Mortality from coronary heart disease in subjects with type 2 diabetes and in nondiabetic subjects with and without prior myocardial infarction. $N$ Engl J Med 1998; 339: 229-234.

5. Vaccaro O, Eberly LE, Neaton JD, Yang L, Riccardi G, Stamler J. Impact of diabetes and previous myocardial infarction on long-term survival: 25-year mortality follow-up of primary screenees of the Multiple Risk Factor Intervention Trial. Arch Intern Med 2004; 164:
$1438-1443$.

6. Miettinen H, Lehto S, Salomaa V, Mahonen M, Niemela M, Haffner SM, et al. Impact of diabetes on mortality after the first myocardial infarction: The FINMONICA Myocardial Infarction Register Study Group. Diabetes Care 1998; 21: 69-75.

7. Abbott RD, Donahue RP, Kannel WB, Wilson PW. The impact of diabetes on survival following myocardial infarction in men vs women: The Framingham Study. JAMA 1988; 260: 3456-3460.

8. Takagi S, Saitoh S, Shimamoto K. Long-term prognosis for diabetics in general population of two rural communities in Japan: A prospective eighteen-year follow-up study. J Jpn Diabetes Society 1998; 41: $257-265$.

9. Tominaga M, Eguchi H, Manaka H, Igarashi K, Kato T, Sekikawa A. Impaired glucose tolerance is a risk factor for cardiovascular disease, but not impaired fasting glucose: The Funagata Diabetes Study. Diabetes Care 1999; 22: 920-924.

10. Deirdre B, James M, Denis M, Samer N, Reubin A, David N, et al. Impaired glucose tolerance, but not impaired fasting glucose, is associated with increased level of coronary heart disease risk factors. Diabetes 2004; 53: 2095-2100.

11. Qing Q, Johan E, Pekka J, Jaakko T. Predictive properties of impaired glucose tolerance for cardiovascular risk are not explained by the development of overt diabetes during follow-up. Diabetes Care 2003; 26: 2910-2914.

12. Kataoka Y, Yasuda S, Morii I, Otsuka Y, Kawamura A, Miyazaki S. Quantitative coronary angiographic studies of patients with angina pectoris and impaired glucose tolerance. Diabetes Care 2005; 28: $2217-2222$.

13. Horimoto M, Hasegawa A, Ozaki T, Takenaka T, Igarashi K, Inoue $\mathrm{H}$. Independent predictors of the severity of angiographic coronary atherosclerosis: The lack of association between impaired glucose tolerance and stenosis severity. Atherosclerosis 2005; 182: $113-$ 119.

14. Austen WG, Edwards JE, Frye RL, Gensini GC, Gott VL, Grifith LSC, et al. AHA Committee Report: A reporting system on patients evaluated for coronary artery disease: Report of the Ad Hoc Committee for Grading of Coronary Artery Disease, Council on Cardiovascular Surgery, American Heart Association. Circulation 1975; 51: $5-40$.

15. Friedewald WT, Levy RI, Fredrickson DS. Estimation of the concentration of low-density lipoprotein cholesterol in plasma, without use of the preparative ultracentrifuge. Clin Chem 1972; 18: 499-502.

16. Expert Committee on the Diagnosis and Classification of Diabetes Mellitus. Report of the Expert Committee on the Diagnosis and Classification of Diabetes Mellitus. Diabetes Care 1997; 20: 1183-1197.

17. Oimatsu H, Saitoh S, Ura N, Shimamoto K. A practical index for evaluation of insulin resistance. J Jpn Diabetes Society 2000; 43: $205-213$.

18. Dortimer AC, Shenoy PN, Shiroff RA, Leaman DM, Babb JD, Liedtke AJ, et al. Diffuse coronary artery disease in diabetic patients: Fact or fiction? Circulation 1978; 57: 133-136.

19. Kip KE, Faxon DP, Detre KM, Yeh W, Kelsey SF, Currier JW. Coronary angioplasty in diabetic patients: The National Heart, Lung, and Blood Institute Percutaneous Transluminal Coronary Angioplasty Registry. Circulation 1996; 94: 1818-1825.

20. Satoh H, Terada H, Uehara A, Katoh H, Matsunaga M, Yamazaki K, et al. Post challenge hyperinsulinaemia rather than hyperglycemia is associated with the severity of coronary artery disease in patients without a previous diagnosis of diabetes mellitus. Heart 2005; 91: $731-736$.

21. Takezako T, Saku K, Zhang B, Shirai K, Arakawa K. Insulin resistance and angiographical characteristics of coronary atherosclerosis. Jpn Circ J 1999; 63: 666-673.

22. Fujiwara T, Saitoh S, Takeuchi H, Isobe T, Miura T, Shimamoto K, et al. Development and progression of atherosclerotic disease in relation to insulin resistance and hyperinsulinemia. Hypertens Res 2005; 28: $665-670$.

23. Kwon K, Choi D, Koo BK, Ryu SK. Decreased insulin sensitivity is associated with the extent of coronary artery disease in patients with angina. Diabetes Obes Metab 2005; 7: 579-585.

24. Hikita N, Tuchihashi K, Nakata T, Ura N, Shimamoto K. Involvement of glucose metabolism abnormalities and insulin resistance in atherosclerotic coronary artery disease: Semiquantitative coronary angiography study. J Cardiol 1998; 32: 291-300.

25. Nemes A, Lengyel C, Forster T, Varkonyi TT, Takacs R, Nagy I, et al. Coronary flow reserve, insulin resistance and blood pressure response to standing in patients with normoglycaemia: Is there a relationship? Diabet Med 2005; 22: 1614-1618.

26. Zhang XF, Attia J, D'este K, Yu XH, Wu XG. Prevalence and magnitude of classical risk factors for coronary heart disease in a cohort 
of 4400 Chinese steelworkers over 13.5 years follow-up. Eur $J$ Cardiovasc Prev Rehabil 2004; 11: 113-120.

27. Mora S, Yanek LR, Moy TF, Fallin MD, Becker LC, Becker DM, et al. Interaction of body mass index and framingham risk score in predicting incident coronary disease in families. Circulation 2005; 111: $1871-1876$.

28. Nasir K, Campbell CY, Santos RD, Roguin A, Braunstein JB,
Carvalho JA. The association of subclinical coronary atherosclerosis with abdominal and total obesity in asymptomatic men. Prev Cardiol 2005; 8: 143-148.

29. Nishio K, Fukui T, Tsunoda F, Kawamura K, Itoh S, Konno N, et al. Insulin resistance as a predictor for restenosis after coronary stenting. Int J Cardiol 2005; 103: 128-134. 